\title{
DETERMINAÇÃO INDIRETA DE N-TOTAL EM PLANTAS POR ESPECTROMETRIA DE ABSORÇÃO ATÔMICA COM CHAMA EMPREGANDO UMA MINI-COLUNA DE AgCl$_{(s)}$
}

\author{
Josiane Meire Tolotti Carneiro*; Elias Ayres Guidetti Zagatto; Ivanildo Luiz de Mattos; Denise \\ Melo \\ Lab. de Química Analítica - USP/CENA, C.P. 96 - CEP: 13400-970 - Piracicaba, SP. \\ *Autor correspondente <josiane@cena.usp.br>

\begin{abstract}
RESUMO: Um sistema de análises químicas por injeção em fluxo empregando uma mini-coluna de $\mathrm{AgCl}_{(\mathrm{s})}$ é proposto para a determinação de nitrogênio total em plantas por espectrometria de absorção atômica com chama. O método se baseia na remoção de íons $\mathrm{Ag}^{+}$por amônia com formação do complexo diamino argentato(I) e posterior direcionamento da amostra a um espectrômetro de absorção atômica onde prata é monitorada. Nitrogênio foi determinado em sete amostras de referência (três replicatas) com precisão e exatidão comparáveis às do procedimento condutimétrico. O sistema é estável, apresentando apenas pequenas variações em sensibilidade $(<2 \%)$ durante períodos de operação de 4 horas. A velocidade analítica é de aproximadamente $100 \mathrm{~h}^{-1}$ e a repetibilidade das medidas é satisfatória (desvio padrão relativo em geral $<0,02$ ).

Palavras-chave: N-total, análise por injeção em fluxo, espectrometria de absorção atômica, mini-coluna
\end{abstract}

\section{INDIRECT DETERMINATION OF N-TOTAL IN PLANT MATERIALS BY FLAME ATOMIC ABSORPTION SPECTROMETRY INVOLVING A AgCl $(\mathrm{s})$ MINI-COLUMN}

\begin{abstract}
A flow-injection system with a $\mathrm{AgCl}_{(\mathrm{s})}$ mini-column is proposed for the determination of total nitrogen in plant digests by flame atomic absorption spectrometry. The method is based on $\mathrm{Ag}^{+}$removal by ammonia through the formation of the diammine argentate(I) complex and further monitoring of the released $\mathrm{Ag}^{+}$. Nitrogen was determined in seven reference samples, in three replicates, with precision and accuracy comparable to the conductimetric procedure. The system is robust, and only slight sensitivity variations $(<2 \%)$ have been noted during extended $(4 \mathrm{~h})$ operating periods. Sampling rate is about $100 \mathrm{~h}^{-1}$ and measurement repeatability is fair (relative standard deviation usually $<0.02$ ).

Key words: N-total, flow-injection analysis, atomic absorption spectrometry, mini-column
\end{abstract}

\section{INTRODUÇÃO}

O desenvolvimento e/ou aprimoramento de métodos para determinação de nitrogênio total em materiais vegetais torna-se necessário devido à importância do controle deste nutriente, em função principalmente de seu papel no metabolismo de aminoácidos e proteínas envolvendo processos enzimáticos e assimilações através de reações de oxiredução (Dougall \& Estaba, 1980). A maioria dos métodos para determinação de nitrogênio total requer a transformação de todas as formas nitrogenadas a amônio e, neste contexto, o método Kjeldahl de digestão desenvolvido em 1883 (Morries, 1983; Jones Jr, 1987) tem sido o mais utilizado para a análise de materiais vegetais.

Íons amônio podem ser quantificados por potenciometria (Shen et al., 1997), titulação (Ohlweiler, 1976) ou espectrofotometria utilizando reagente de Nessler (Dorich \& Nelson, 1983), salicilato de sódio (Bremner \& Mulvaney, 1982), etc, em procedimentos que freqüentemente requerem etapas de difusão gasosa (Oms et al., 1996) ou destilação (Jones Jr, 1987). Neste sentido, deve-se ressaltar que o método espectrofotométrico do azul de indofenol (Searle, 1984) não envolve separação de fases e tem sido um dos mais utilizados, apesar da carcinogenicidade dos reagentes empregados.

A determinação de nitrogênio amoniacal em sistemas de análises em fluxo tem empregado principalmente potenciometria, espectrofotometria UV-vis e condutimetria, envolvendo em geral difusão gasosa (Faria \& Pasquini, 1991; Reis et al., 1997); métodos espectrofotométricos diretos tais como o do azul de indofenol (Searle, 1984; Van Staden \& Taljaard, 1997) tem sido também implementados em sistemas de análises em fluxo.

Recentemente, tem se verificado uma tendência à incorporação de reativos sólidos pouco solúveis aos sistemas de análises em fluxo (Mottola, 1989; Teshima \& Sakai, 1999), em função da crescente necessidade por métodos rápidos e sensíveis. A cinética reacional é favorecida porque a concentração do reagente imobilizado não é limitada por efeitos de solubilidade (Perez-Bendito \& Silva, 1988).

Neste sentido, uma estratégia para determinação indireta de glicina em formulações farmacêuticas por espectrometria de absorção atômica em sistemas de 
análises por injeção em fluxo foi proposta por Calatayud \& Garcia Mateo (1991). Uma mini-coluna de carbonato de cobre imobilizado era inserida no percurso analítico do sistema. Após a injeção da amostra, a mesma fluía através da mini-coluna e os íons $\mathrm{Cu}^{2+}$ eram removidos devido à formação do complexo Cu-glicina, seguindo para um espectrômetro de absorção atômica, onde eram determinados. O sinal analítico era proporcional ao teor de glicina na amostra injetada.

Similarmente, um procedimento para imobilização de reagentes em materiais poliméricos foi descrito para a determinação indireta de isoniazida por espectrometria de absorção atômica (Zamora et al., 1992). O fármaco era oxidado com dióxido de manganês imobilizado em resina de poliester, e as medidas de $\mathrm{Mn}^{2+}$ liberado após a oxidação eram proporcionais ao teor de isoniazida na amostra.

A polimerização com poliéster de cadeia linear insaturada foi também aplicada para imobilização de carbonato de cobre visando a determinação indireta de glicina por espectrometria de absorção atômica (Garcia Mateo \& Calatayud, 1993).

Em 1995, Rivas \& Calatayud desenvolveram um método para a determinação de ácido salicílico utilizando o carbonato de cobre em fase sólida incorporado em resina de poliéster. A determinação era feita após a interação da amostra com o carbonato de cobre, e os íons $\mathrm{Cu}^{2+}$ liberados eram monitorados por espectrometria de absorção atômica.

Outras alternativas têm sido propostas com relação ao emprego de reagentes sólidos em conexão com sistemas de análises em fluxo e espectrometria de absorção atômica (Hassan, 1984; Teshima \& Sakai, 1999). Neste contexto, o emprego de $\mathrm{AgCl}$ imobilizado foi descrito por Esmadi et al. (1990), que determinaram cianeto, tiossulfato e amônio. A amostra era injetada e fluía através da mini-coluna contendo cloreto de prata imobilizado em pérolas de vidro, liberando íons $\mathrm{Ag}^{+}$em direção ao nebulizador de um espectrômetro de absorção atômica.

Similarmente, uma mini-coluna de $\mathrm{AgCl}_{(\mathrm{s})}$ foi empregada para a determinação de amônio (Icardo et al., 1999) em amostras de fertilizantes e de formulações farmacêuticas, porém o procedimento envolvia uma etapa de separação prévia de interferentes. Neste mesmo ano, Carneiro et al. (2000) adaptaram este procedimento à análise de digeridos Kjeldahl. O método proposto envolvia detecção espectrofotométrica e se baseava na interação do complexo diamino argentato(I) com vermelho de bromopirogalol (VBP) e o-fenantrolina (phen) resultando num complexo ternário colorido monitorado a $635 \mathrm{~nm}$. Neste sistema, a solução fluindo através da mini-coluna apresentava uma concentração apropriada de cloreto de sódio de forma a minimizar o lixiviamento de $\mathrm{AgCl}$ da minicoluna por efeito do íon comum.

Visando simplificação, um espectrômetro de absorção atômica pode ser utilizado de forma a não serem mais necessários os reagentes VBP e phen, o ajuste de $\mathrm{pH}$ final, e alguns componentes do módulo de análise.
O objetivo deste trabalho foi desenvolver um sistema de análises por injeção em fluxo empregando uma mini-coluna com reagente pouco solúvel $(\mathrm{AgCl})$ e explorando a espectrometria de absorção atômica com chama ar-acetileno para a determinação em larga escala de $\mathrm{N}$-total em materiais vegetais.

\section{MATERIAL E MÉTODOS}

Todas as soluções foram preparadas com reativos de grau analítico e água desionizada.

A solução-padrão estoque $\left(1,333 \mathrm{~g} \mathrm{~L}^{-1} \mathrm{~N}\right)$ foi preparada a partir da dissolução de $1,572 \mathrm{~g}\left(\mathrm{NH}_{4}\right)_{2} \mathrm{SO}_{4} \mathrm{em}$ aproximadamente $50 \mathrm{~mL}$ com água e completando-se 0 volume até $250 \mathrm{~mL}$ de água. As soluções-padrão de trabalho eram preparadas semanalmente na faixa de 26,6 a $159,6 \mathrm{mg} \mathrm{L}^{-1} \mathrm{~N}$, o que correspondia a uma faixa de 1,00 a $6,00 \%(\mathrm{~m} / \mathrm{m}) \mathrm{N}$ na matéria seca; $1,00 \mathrm{~mL}$ da solução estoque diluído para $50 \mathrm{~mL}$ de $0,9 \mathrm{~mol} \mathrm{~L}^{-1} \mathrm{H}_{2} \mathrm{SO}_{4}$ correspondia a um teor de $1,00 \%(\mathrm{~m} / \mathrm{m}) \mathrm{N}$ na matéria seca. Em função da acidez dos digeridos Kjeldhal e das soluções-padrão de trabalho, uma solução $0,9 \mathrm{~mol} \mathrm{~L}^{-1}$ $\mathrm{H}_{2} \mathrm{SO}_{4}$ foi empregada como fluxo transportador.

A solução $0,25 \mathrm{~mol} \mathrm{~L}^{-1} \mathrm{Na}_{2} \mathrm{~B}_{4} \mathrm{O}_{7}$ era preparada em $0,9 \mathrm{~mol} \mathrm{~L}^{-1} \mathrm{NaOH}$ e $5,0 \times 10^{-3} \mathrm{~mol} \mathrm{~L}^{-1} \mathrm{Cl}^{-}$, a partir da solução estoque de $1,0 \mathrm{~mol} \mathrm{~L}^{-1} \mathrm{NaCl}$.

As soluções contendo espécies químicas interferentes em potencial foram preparadas nas concentrações de $20 \mathrm{mg} \mathrm{L}^{-1} \mathrm{Cl}, 60 \mathrm{mg} \mathrm{L}^{-1} \mathrm{P}_{-} \mathrm{PO}_{4}, 20 \mathrm{mg}$ $\mathrm{L}^{-1} \mathrm{Ca}, 10 \mathrm{mg} \mathrm{L}^{-1} \mathrm{Mg}, 10 \mathrm{mg} \mathrm{L}^{-1} \mathrm{Fe}(\mathrm{III}), 4,0 \mathrm{~mL} \mathrm{~L}^{-1} \mathrm{Al}, 2,0$ $\mathrm{mg} \mathrm{L}^{-1} \mathrm{Mn}(\mathrm{II}), 0,5 \mathrm{mg} \mathrm{L}^{-1}$ Cu e 2,0 $\mathrm{mol} \mathrm{L}^{-1} \mathrm{~S}_{-} \mathrm{SO}_{4}$.

A solução digestora foi preparada misturando-se $350 \mathrm{~mL}$ de $\mathrm{H}_{2} \mathrm{O}_{2}$ a $30 \%, 14,0 \mathrm{~g} \mathrm{Li}_{2} \mathrm{SO}_{4}$ e $0,42 \mathrm{~g}$ de selênio em pó em um copo de $1000 \mathrm{~mL}$ resfriado em banho de gelo. Em seguida, adicionaram-se lenta e cuidadosamente, $420 \mathrm{~mL}$ de $\mathrm{H}_{2} \mathrm{SO}_{4}$ concentrado, mantendo-se agitação e evitando-se 0 aquecimento. A mistura digestora foi conservada sob refrigeração (Parkison \& Allen, 1975).

As amostras eram mineralizadas por meio de digestão sulfúrica, de forma similar à descrita por Parkison \& Allen (1975), porém envolvendo 0,4 g de amostra vegetal seca e moída.

A instrumentação compreendia um espectrômetro de absorção atômica modelo Perkin-Elmer 503 equipado com lâmpada de catodo oco de prata, operado segundo recomendações do fabricante para máxima sensibilidade com chama ar-acetileno, e conectado a um registrador potenciométrico modelo Kipp \& Zonen 111; uma bomba peristáltica Ismatec IPC-08 com tubos de Tygon de diferentes diâmetros internos para o bombeamento das soluções; e um injetor proporcional (Krug et al., 1986). Tubos de polietileno de $0,8 \mathrm{~mm}$ de diâmetro interno e conectores de acrílico foram empregados para a montagem do módulo de análise.

Como a granulação das partículas de $\mathrm{AgCl}$ era um parâmetro crítico para o projeto do sistema, as minicolunas foram confeccionadas após definir-se um padrão 
por meio da utilização de peneira com abertura de 0,35 mm (Carneiro et al., 1999). Deve-se salientar que a utilização de partículas muito grossas resultava em perda de sensibilidade, ao passo que partículas muito finas levavam a um aumento do lixiviamento de $\mathrm{AgCl}$ e a uma maior compactação da mini-coluna aumentando a pressão hidrodinâmica no sistema. As mini-colunas foram então preparadas preenchendo-se, com auxílio de seringa, um tubo de Tygon de 3,2 $\mathrm{mm}$ de diâmetro interno com $5 \mathrm{~cm}$ de comprimento com aproximadamente $1 \mathrm{~g}$ de cloreto de prata previamente peneirado (malha $=0,35 \mathrm{~mm}$ ).

O sistema mostrado na Figura 1 foi empregado para avaliação das condições de operação e determinação de íons prata (determinação indireta de nitrogênio). As soluçõespadrão $(1,00$ a $6,00 \% \mathrm{~m} / \mathrm{m} \mathrm{N}$, base em matéria seca) injetadas eram transportadas por um fluxo ácido $\left(0,9 \mathrm{~mol} \mathrm{~L}^{-1}\right.$ $\mathrm{H}_{2} \mathrm{SO}_{4}-1,2 \mathrm{~mL} \mathrm{~min}^{-1}$ ) convergente com o reagente $\mathrm{Na}_{2} \mathrm{~B}_{4} \mathrm{O}_{7}$ $+\mathrm{NaOH}+\mathrm{NaCl}\left(3,8 \mathrm{~mL} \mathrm{~min}^{-1}\right)$ e homogeneizada no reator B $(50 \mathrm{~cm})$. A amostra fluia através da mini-coluna na qual ocorria a reação de amônia com íons prata(I) com formação do complexo solúvel diamino argentato(I). A seguir, a zona de amostra processada seguia em direção ao nebulizador do espectrômetro de absorção atômica onde 0 monitoramento de prata era feito a $328,1 \mathrm{~nm}$.

Os parâmetros aplicados ao sistema proposto (granulação das partículas de $\mathrm{AgCl}$, alcalinidade do meio reacional, natureza e concentração da solução-tampão, vazões, intensidade iônica no ambiente reacional, temperatura, comprimento da alça de amostragem e da bobina de reação, concentração de íons cloreto e presença dos íons interferentes em potencial) foram selecionados de acordo com o método para determinação de $\mathrm{N}$-total por espectrofotometria proposto por Carneiro et al. (1999). Para avaliação de seletividade, soluções individuais com diferentes teores de $\mathrm{N}$ e de cada espécie química potencialmente interferente (concentrações acima especificadas) deveriam ser empregadas. Para se evitar o preparo de um conjunto de soluções excessivamente grande, foi conduzido um experimento no qual cada solução-padrão convergia com cada solução contendo um interferente em potencial sob vazões similares, a mescla sendo dirigida para a alça de amostragem.

Após o dimensionamento do sistema, suas principais características analíticas foram avaliadas visando sua aplicação a análises em larga escala.

\section{RESULTADOS E DISCUSSÃO}

$\mathrm{O}$ controle de $\mathrm{pH}$ na mini-coluna é de fundamental importância, pois pequenas variações $( \pm 0,3)$ causavam interferência pronunciada (até $30 \%$ ) na quantidade de íons prata deslocados comprometendo ainda a estabilidade do complexo diamino argentato(I) formado. Os melhores resultados foram obtidos para $\mathrm{pH}$ em torno de 11,0 e 11,5, o qual era obtido utilizando-se $0,25 \mathrm{~mol} \mathrm{~L}^{-1} \mathrm{Na}_{2} \mathrm{~B}_{4} \mathrm{O}_{7}$ e 0,9 mol L-1 $\mathrm{NaOH}$ em $\mathrm{R}$ (Figura 1 ). Em experimentos preliminares, foi verificado que, para valores de $\mathrm{pH}$

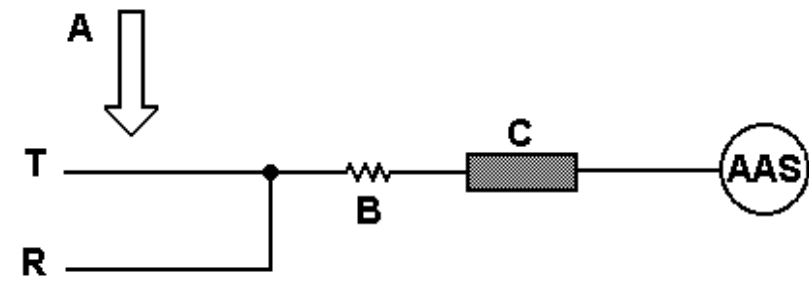

Figura 1 - Diagrama de fluxos do sistema proposto. $A=$ amostra injetada por meio de uma alça de amostragem de 5,0 $\mathrm{cm}$ (ca $30 \mu \mathrm{L}) ; \mathrm{T}$ = solução transportadora $\left(0,9 \mathrm{~mol} \mathrm{~L}^{-1}\right.$ $\mathrm{H}_{2} \mathrm{SO}_{4}$ ) a $1,2 \mathrm{~mL} \mathrm{~min}^{-1} ; \mathrm{R}=$ solução $0,25 \mathrm{~mol} \mathrm{~L}^{-1} \mathrm{Na}_{2} \mathrm{~B}_{4} \mathrm{O}_{7}$ $+0,9 \mathrm{~mol} \mathrm{~L}^{-1} \mathrm{NaOH}+5,0 \times 10^{-3} \mathrm{~mol} \mathrm{~L}^{-1} \mathrm{Cl}^{-1}$ a $3,8 \mathrm{~mL} \mathrm{~min}^{-1}$; $\mathrm{C}=$ mini-coluna de $\mathrm{AgCl}_{(\mathrm{s})} ; \mathrm{B}=$ reator tubular helicoidal $(50 \mathrm{~cm}) ; \mathrm{AAS}=$ espectrômetro de absorção atômica.

superiores a 12,0 ocorria a formação de um composto negro, provavelmente óxido de prata, ao passo que sob menores alcalinidades ocorria perda pronunciada de sinal devido à predominância de amônio sobre amônia, prejudicando a formação do complexo diamino argentato(I).

As concentrações de cloreto em $\mathrm{R}$ também foram definidas avaliando-se as melhores condições de estabilidade do sistema bem como de sensibilidade e repetibilidade analíticas. Obtiveram-se melhores resultados quando esta concentração se situou ao redor de 5,0 x $10^{-3} \mathrm{~mol} \mathrm{~L}^{-1} \mathrm{Cl}^{-}$. Sem adição de cloreto de sódio em $\mathrm{R}, \mathrm{a}$ operação do sistema se inviabilizava em função da elevada linha base; nestas condições, a mini-coluna era totalmente lixiviada por solubilização em aproximadamente 4 horas. Por outro lado, quando o teor de cloreto em $\mathrm{R}$ era excessivo $\left(>1,0 \times 10^{-2} \mathrm{~mol} \mathrm{~L}^{-1}\right)$, o sinal analítico sofria redução pronunciada em função provavelmente da competição dos íons cloreto com os ligantes diamino pelo cátion prata com formação do precipitado $\mathrm{AgCl}$. Observouse ainda que, apesar da pequena redução (ca $15 \%$ em sinal analítico, a presença de cloreto em $\mathrm{R}$ a uma concentração de $5,0 \times 10^{-3} \mathrm{~mol} \mathrm{~L}^{-1}$ melhorou significativamente a linearidade da curva analítica diminuindo o lixiviamento de $\mathrm{AgCl}$.

O comprimento da alça de amostragem define 0 volume de amostra introduzido no sistema, e foi selecionado como $5 \mathrm{~cm}$ visando uma alta diluição no ponto de confluência e conseqüente melhoria nas condições de tamponização. Para menores volumes, a repetibilidade das medidas se deteriorava, sendo o desvio padrão relativo das medidas estimado como $>10 \%$. Por outro lado, o controle de $\mathrm{pH}$ se tornava mais difícil para maiores volumes injetados, em função do conseqüente menor grau de diluição da amostra, considerando-se ainda que com o aumento da diluição diminuem as eventuais variações de acidez entre amostras de um mesmo lote. Ainda, a linearidade da curva analítica era comprometida para maiores volumes de amostra. Com $30 \mu \mathrm{L}$ de volume injetado, uma bobina de reação (B) de $50 \mathrm{~cm}$ de comprimento foi suficiente para a homogeneização adequada da zona de amostra, e a repetibilidade das medidas se mostrou adequada. 
O tempo decorrido durante a passagem do analito pela mini-coluna de $\mathrm{AgCl}$ não apresentou relação com a sensibilidade e repetibilidade analíticas, demonstrando que os resultados são pouco dependentes da cinética reacional. Neste sentido, experimentos adicionais envolvendo desligamento da bomba peristáltica revelaram que as reações químicas se completavam no percurso analítico, desde que não foram verificadas variações nos valores de absorbância.

O sistema proposto é praticamente não influenciado por variações de temperatura. De fato, experimentos conduzidos imergindo-se os frascos de reagentes, a mini-coluna e o reator em banho de temperatura controlada revelaram modificações em sinais analíticos menores do que $10 \%$ para variações pronunciadas de temperatura $\left(5-45^{\circ} \mathrm{C}\right)$. O sistema pode então ser operado sob temperatura ambiente, mantida sob refrigeração (ar condicionado).

Com relação às espécies químicas potencialmente interferentes, nenhum dentre os íons testados interferiu nos resultados obtidos, demonstrando que o procedimento pode ser aplicado às análises de plantas, alimentos e similares sem restrições.

$\mathrm{Na}$ Figura 2, observa-se o registro da etapa de calibração e a correspondente curva analítica, a qual pode ser expressa pela equação:

$h=-0,0049+0,01149[N]+0,00095[N]^{2}(n=6, r=0,9997)$

onde: $\mathrm{h}$ = altura do pico registrado, em absorbância; $[\mathrm{N}]$ = concentração de $\mathrm{N}$-total na amostra em $\mathrm{m} / \mathrm{m}$ (base: matéria seca).

O sistema proposto mostrou-se estável, e somente pequenas variações em sensibilidade $(<2 \%)$ foram observadas após o seu emprego por longos (4 horas) períodos de operação. A velocidade analítica é de ca 100 determinações por hora o que corresponde a $36,2 \mathrm{mg}$ de tetraborato de sódio consumidos por determinação. A linha base mantém-se estável, com derivas em geral $<0,01 \mathrm{~A}$ por hora de operação, e a repetibilidade das medidas é satisfatória: desvios padrão relativos dos resultados referentes a 10 injeções sucessivas de duas amostras com 1,83 e $3,52 \% \mathrm{~m} / \mathrm{m} \mathrm{N}$ foram estimados como 5,1 e $0,6 \%$, respectivamente.

Uma mini-coluna pode ser utilizada para mais de 2000 determinações, podendo ainda ser retirada, estocada e reutilizada sem a necessidade de recondicionamento.

A exploração da abordagem de zonas coalescentes (Bergamin Filho et al., 1978) é recomendada para a análise de grandes lotes de amostras. Com esta estratégia, a amostra e o reagente são introduzidos em fluxos transportadores quimicamente inertes e convergentes, os quais atuam também como solução de limpeza. Desta forma, apenas o fluxo transportador principal atinge 0 instrumento durante a maior parte do ciclo analítico. A entrada contínua de sódio no nebulizador é então minimizada, e o consumo de reagente é diminuído.
A exatidão analítica foi confirmada comparando-se os resultados obtidos com os valores relatados para materiais de referência e com os resultados obtidos com o método condutimétrico (Reis et al., 1997). Análise estatística (teste t) dos dados da TABELA 1 não indica diferenças significativas entre métodos a $95 \%$.

\section{CONCLUSÕES}

A combinação de mini-coluna de cloreto de prata, sistemas de análises em fluxo e espectrometria de absorção atômica permitiu o desenvolvimento de um método para a determinação de $\mathrm{N}$-total em plantas, alimentos e similares apresentando características favoráveis, tais como simplicidade do sistema, alta velocidade analítica, economia de reagentes, robustez e estabilidade. Reagentes cromogênicos tóxicos e/ou carcinogênicos, bem como etapas de difusão gasosa e/ ou destilação, rotineiramente empregadas para esta determinação, não foram requeridos.

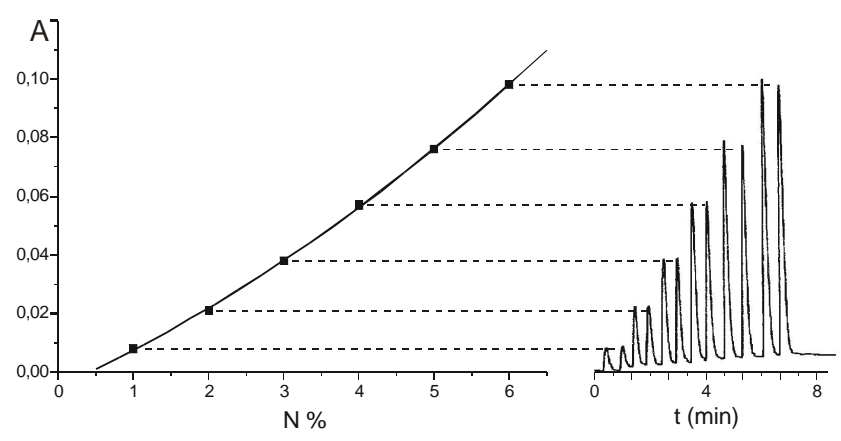

Figura 2 - Registro da etapa de calibração e a correspondente curva analítica.

TABELA 1 - Resultados comparativos. Dados determinados pelo método proposto (AAS) e por condutimetria (Reis et al., 1997).

\begin{tabular}{|c|c|c|c|}
\hline Amostra & AAS & Condutimetria & $\begin{array}{c}\text { Valor } \\
\text { certificado }\end{array}$ \\
\hline
\end{tabular}

$\% \mathrm{~N} \mathrm{~m} / \mathrm{m}$ na matéria seca

\begin{tabular}{|c|c|c|c|}
\hline Soja & $6,01 \pm 0,03$ & $6,11 \pm 0,02$ & - \\
\hline Tabaco & $2,55 \pm 0,05$ & $2,60 \pm 0,03$ & - \\
\hline Pinus & $1,91 \pm 0,03$ & $1,90 \pm 0,02$ & - \\
\hline Café & $2,71 \pm 0,02$ & $2,65 \pm 0,02$ & - \\
\hline Feijão & $2,85 \pm 0,01$ & $2,89 \pm 0,02$ & - \\
\hline Arroz & $1,59 \pm 0,03$ & $1,55 \pm 0,03$ & - \\
\hline $\begin{array}{l}\text { Folha de } \\
\text { soja }\end{array}$ & $2,34 \pm 0,05$ & $2,42 \pm 0,06$ & - \\
\hline Cotonnier* & $3,79 \pm 0,03$ & $3,85 \pm 0,02$ & $3,821 \pm 0,153$ \\
\hline Pecher* & $4,39 \pm 0,01$ & $4,31 \pm 0,02$ & $4,215 \pm 0,237$ \\
\hline
\end{tabular}

* Amostras provenientes (1981) do Instituto ORSTOM, Paris. 


\section{AGRADECIMENTOS}

À FAPESP, CNPq e FINEP/PRONEX pelo apoio financeiro recebido e a B.F. Reis pelos comentários construtivos.

\section{REFERÊNCIAS BIBLIOGRÁFICAS}

BERGAMIN FILHO, H.; ZAGATTO, E.A.G.; REIS, B.F. Merging zones in flow injection analysis: Part I. Double proportional injector and reagent consumption. Analytica Chimica Acta, v.101, p.17-23, 1978.

BREMNER, J.M.; MULVANEY, C.S. Nitrogen-total. In: PAGE, A.L. (Ed.) Methods of soil analysis. Madison: ASA, 1982. pt.2, p.595-624.

CALATAYUD, J.M.; GARCIA MATEO, J.V. High-pressure flowinjection assembly. Indirect determination of glycine by atomic absorption spectrometry. The Analyst, v.116, p.327-329, 1991.

CARNEIRO, J.M.T.; SARTINI, R.P.; ZAGATTO, E.A.G. Spectrophotometric determination of total nitrogen in plant materials using a flow-injection system with a $\mathrm{AgCl}_{(\mathrm{s})}$ reactor. Analytica Chimica Acta, v.416, p.185-190, 2000.

DORICH, R.A.; NELSON, D.W. Direct colorimetric measurement of ammonium in potassium chloride extracts of soils. Soil Science Society of America Journal, v.47, p.833-836, 1983.

DOUGALL, D.K.; ESTABA, D.J. Nutrition and metabolism. In: STABA, E.J. (Ed.) Plant tissue culture as source of biochemicals. Boca Raton: CRS Press, 1980. p.21-58.

ESMADI, F.T.; KHAROAF, M.; ATTIYAT, A.S. Indirect atomicabsorption spectrophotometric determination of ammonia, thiosulphate and cyanide in an unsegmented flow system. Analytical Letters, v.23, p.1069-1086, 1990.

FARIA, L.C.; PASQUINI, C. Flow injection determination of inorganic forms of nitrogen by gas diffusion and conductimetry. Analytica Chimica Acta, v.245, p.183-190, 1991.

GARCIA MATEO, J.V.; CALATAYUD, J.M. Entrapped copper(II) carbonate for indirect determination of glycine by flow injection atomic-absorption spectrometry. Analytica Chimica Acta, v.274, p.275-281, 1993.

HASSAN, S.S.M. Organic analysis using atomic absorption spectrometry. Chichester: Ellis Horwood, 1984.

ICARDO, M.C.; TORRÓ, I.G.; ZAMORA, L.L.; CALATAYUD, J.M. Flow spectrophotometric determination of ammonium ion. Analytica Chimica Acta, v.398, p.311-318, 1999.

JONES Jr, J.B. Kjeldahl nitrogen determination-What's in a name. Journal of Plant Nutrition, v.10, p.1675-1682, 1987.

KRUG, F.J.; BERGAMIN FILHO, H.; ZAGATTO, E.A.G. Commutation in flow injection analysis. Analytica Chimica Acta, v.179, p.103-18, 1986.
MORRIES, P. A century of Kjeldahl (1883-1983). Journal of the Association of Public Analysts, v.21, p.53-58, 1983.

MOTTOLA, $H$. Strategies for the use of immobilized reagents in continuous-flow situations. Química Analítica (Espanha), v.8, p.119, 1989.

OHLWEILER, O.A. Química analítica quantitativa. 2.ed. Rio de Janeiro: Livros Técnicos e Científicos, 1976. v.2, cap.18, p.452-494: Titulometria de neutralização.

OMS, M.T.; CERDÀ, A.; CLADERA, A.; CERDÀ, V.; FORTEZA, R. Gas diffusion techniques coupled sequential injection analysis for selective determination of ammonium. Analytica Chimica Acta, v.318, p.251-260, 1996.

PARKISON, J.A.; ALLEN, S.E. A wet oxidation procedure suitable for the determination of nitrogen and mineral nutrients in biological material. Communications in Soil Science and Plant Analysis, v.6, p.1-11, 1975.

PEREZ-BENDITO, D.; SILVA, M. Kinetic methods in analytical chemistry. Chichester: Ellis Horwood, 1988. 330p.

REIS, B.F.; KRUG, F.J.; VIEIRA, J.A.; GINÉ, M.F. Development of a flow injection system with two analytical paths for ammonium determination in soil extracts by conductometry. Journal of the Brazilian Chemical Society, v.8, p.523-528, 1997.

RIVAS, G.A.; CALATAYUD, J.M. FIA-AAS determination of salicylic acid by a solid-phase reactor of copper carbonate incorporated in polyester resin beads. Talanta, v.42, p.1285-1289, 1995.

SEARLE, P.L. The Berthelot or indophenol reaction and its use in the analytical chemistry of nitrogen. a review. The Analyst, v.109, p.549-568, 1984

SHEN, H.; CARDWELL, T.J.; CATTRALL, R.W. Determination of ammonia in waste water by a differential $\mathrm{pH}$ method using flow injection potentiometry and a nonactin-based sensor. The Analyst, v.122, p.89-93, 1997.

TESHIMA, N.; SAKAI, T. FIA Bibliography (31). Journal of Flow Injection Analysis, v.16, p.91-112, 1999.

VAN STADEN, J.F.; TALJAARD, R.E. Determination of ammonia in water and industrial effluent streams with the indophenol blue method using sequential injection analysis. Analytica Chimica Acta, v.344, p.281-289, 1997.

ZAMORA, L.L.; MATEO, J.V.G.; CALATAYUD, J.M. Entrapment of reagents in polymeric materials. Indirect atomic absorption spectrometric determination of isoniazid by oxidation with manganese dioxide incorporated in polyester resin beads in a flow injection system. Analytica Chimica Acta, v.265, p.81-86, 1992.

$\overline{\text { Recebido em } 13} .03 .00$ 